\title{
Alcohol-based highly conductive polymer for conformal nanocoatings on hydrophobic surfaces toward a highly sensitive and stable pressure sensor
}

\author{
Jung Joon Lee $\mathbb{1}^{1}$, Srinivas Gandla', Byeongjae Lim², Sunju Kang ${ }^{1}$, Sunyoung Kim², Sunjong Lee ${ }^{2}$ and Sunkook Kim
}

\begin{abstract}
Conformal and ultrathin coating of highly conductive PEDOT:PSS on hydrophobic uneven surfaces is essential for resistive-based pressure sensor applications. For this purpose, a water-based poly(3,4-ethylenedioxythiophene) polystyrene sulfonate (PEDOT:PSS) solution was successfully exchanged to an organic solvent-based PEDOT:PSS solution without any aggregation or reduction in conductivity using the ultrafiltration method. Among various solvents, the ethanol (EtOH) solvent-exchanged PEDOT:PSS solution exhibited a contact angle of $34.67^{\circ}$, which is much lower than the value of $96.94^{\circ}$ for the water-based PEDOT:PSS solution. The optimized EtOH-based PEDOT:PSS solution exhibited conformal and uniform coating, with ultrathin nanocoated films obtained on a hydrophobic pyramid polydimethylsiloxane (PDMS) surface. The fabricated pressure sensor showed high performances, such as high sensitivity $\left(-21 \mathrm{kPa}^{-1}\right.$ in the low pressure regime up to $100 \mathrm{~Pa}$ ), mechanical stability (over 10,000 cycles without any failure or cracks) and a fast response time $(90 \mathrm{~ms})$. Finally, the proposed pressure sensor was successfully demonstrated as a human blood pulse rate sensor and a spatial pressure sensor array for practical applications. The solvent exchange process using ultrafiltration for these applications can be utilized as a universal technique for improving the coating property (wettability) of conducting polymers as well as various other materials.
\end{abstract}

\section{Introduction}

Flexible pressure sensors that can transform the physical sense of pressure into meaningful information through a capacitive, resistive, or piezoelectric medium have gained substantial attention due to the significant findings for various promising applications, including rollable touch displays, artificial electronic skin, robotics, and healthcare monitoring $^{1-4}$. Promising routes to improve the sensor performance in terms of good mechanical stability, high sensitivity, high response time, and robustness through

\footnotetext{
Correspondence: Sunjong Lee (sunjong1774@kitech.re.kr) or Sunkook Kim (seonkuk@skku.edu)

${ }^{1}$ Multifunctional Nano Bio Electronics Lab, Department of Advanced Materials and Science Engineering, Sungkyunkwan University, Suwon, Republic of Korea ${ }^{2}$ Research Institute of Sustainable Manufacturing System, Korea Institute of Industrial Technology, Cheonan 31056, Republic of Korea

These authors contributed equally: Jung Joon Lee, Srinivas Gandla
}

newly emerged advanced functional materials have been demonstrated in recent years. Based on various sensing mechanisms, resistive-type pressure sensors have been widely reported to provide additional functionalities to the sensor. Microstructures of elastomer materials have been the key factor in providing rubber-like elastic mechanical behavior to these sensors. However, elastomer materials are hydrophobic, and it becomes difficult to deposit uniform and adherent films of conductive materials. Moreover, the situation becomes worse on uneven surfaces. Although conductive materials, such as metals, can be deposited uniformly over large areas, they are brittle and difficult to apply to flexible devices. Therefore, materials possessing flexibility, high conductivity and good wettability are essential. In this respect, conducting polymers are very suitable due to the characteristics of high flexibility, good

\section{(c) The Author(s) 2020}

(c) (i) Open Access This article is licensed under a Creative Commons Attribution 4.0 International License, which permits use, sharing, adaptation, distribution and reproduction cc) in any medium or format, as long as you give appropriate credit to the original author(s) and the source, provide a link to the Creative Commons license, and indicate if changes were made. The images or other third party material in this article are included in the article's Creative Commons license, unless indicated otherwise in a credit line to the material. If material is not included in the article's Creative Commons license and your intended use is not permitted by statutory regulation or exceeds the permitted use, you will need to obtain permission directly from the copyright holder. To view a copy of this license, visit http://creativecommons.org/licenses/by/4.0/. 
conductivity, large area, mass producibility, solution processability, and so on ${ }^{5-12}$. PEDOT:PSS was chosen for this work because it has the highest conductivity among various conducting polymers. However, commercialized PEDOT: PSS with high conductivity is dispersed in water, so the ability of the water-based PEDOT:PSS solution to coat on hydrophobic elastomeric substrates is limited due to the insufficient wettability of the aqueous solution. Thus, to provide uniform and adhered films, either the substrates undergo various surface treatments to facilitate hydrophilicity or wetting agents (e.g., the Zonyl surfactant) are added to the water-based PEDOT:PSS solution to provide good wettability. In either of these cases, there is always the possibility that the substrate will become oxidized, and the original characteristics will be modified or damaged. In the case of surfactants, the Zonyl surfactant in particular was registered as a regulated material under international regulatory policies, in which perfluorooctane sulfonate and perfluorooctanoic acid containing substantial amounts of fluorine atoms may lead to health issues; therefore, its production was discontinued in $2015^{13-15}$. To overcome this issue, solvent exchange can be a good alternative because the contact angle with a hydrophobic substrate is lower in organic solvents than in water, resulting in better wettability. Several studies have reported exchange of water-based PEDOT:PSS solutions to organic solvents ${ }^{16-19}$. Despite the methods being attractive, the previous results exhibited low conductivity of PEDOT:PSS and involved limited solvents with high b.p. only; thus, the processing is very complicated, and the production scale is insufficient. The limitations pertaining to these methods are presented in Table 1 . Therefore, it is highly essential to find a simple and proper way to improve the coating property without deteriorating the intrinsic properties of the PEDOT:PSS material.

Herein, we propose a facile way to greatly enhance the coating property of the PEDOT:PSS polymer on hydrophobic substrates using a solvent-exchange ultrafiltration method, presenting a solution to all the aforementioned concerns. The method not only exchanges solvents with high b.p. but also solvents with low b.p., such as volatile solvents (alcohols), while retaining the high conductivity of pristine PEDOT:PSS. In addition, the solvent exchange process is fast, universal, and easy to mass produce. Once PEDOT:PSS is dried in a solid state, it does not redisperse back into a solvent in its original state due to coagulation. Therefore, the solvent exchange process was designed to maintain the wet state of PEDOT:PSS until the end to avoid coagulation of PEDOT:PSS for good redispersion in organic solvent. Overall, the EtOH-based PEDOT:PSS solution made by this solvent exchange process exhibited excellent wettability on a hydrophobic PDMS substrate with a micropyramid array compared to other solvents without any additional surfactant. The pressure sensor fabricated using this approach exhibited high sensitivity in the low-pressure regime $\left(-21 \mathrm{kPa}^{-1},<100 \mathrm{~Pa}\right)$ possessing an exceptional uniform and conformal coating without any discontinuity, which is not the case with the waterbased PEDOT:PSS. Moreover, the sensor was robust to extreme loads of cyclic mechanical behavior over 10,000 cycles without any failure or crack formation and had a fast response time of $90 \mathrm{~ms}$, with a maximum load of $1 \mathrm{kPa}$. The pressure sensor was successfully demonstrated as a human blood pulse rate sensor and a spatial pressure

Table 1 Previously reported solvent exchange processes for water-based PEDOT:PSS solutions.

\begin{tabular}{|c|c|c|c|c|}
\hline Method & Solvent & $\begin{array}{l}\text { Stability of } \\
\text { re-dispersion }\end{array}$ & Problems (limitations) & Ref. \\
\hline Mixture \& Evaporation & NMP, DMAC, DMF, etc. & Not verified & $\begin{array}{l}\text {-Limited solvent (High b.p }>150^{\circ} \mathrm{C} \text { ) } \\
\text { coating drying temperature requirement, poor } \\
\text {-Environmentally restricted materials (NMP, DMAC, } \\
\text { DMF) by EU, "REACH" (2018.10.31 Present) }\end{array}$ & 19 \\
\hline Mixture $(3: 1 \mathrm{v} / \mathrm{v})$ & DMSO, DMF, THF & Not verified & -Simple mixing, not solvent exchange & 20 \\
\hline $\begin{array}{l}\text { Synthesis of Alkyl } \\
\text { derivative PEDOT }\end{array}$ & Chloroform, DMF & Good & $\begin{array}{l}\text {-Far less conductive } \\
\text {-Very complex, small production }\end{array}$ & 21 \\
\hline $\begin{array}{l}\text { Synthesis of PEDOT:PSS in } \\
\text { organic solvent }\end{array}$ & Methanol, THF, Toluene, etc. & Moderate & $\begin{array}{l}\text {-Far less conductive } \\
\text {-Complex and complicated }\end{array}$ & 22 \\
\hline Ultrafiltration & $\begin{array}{l}\text { All with high } \partial \mathrm{H}(>19.4) \text { such as } \\
\text { EtOH, EG, etc. }\end{array}$ & Very good & $\begin{array}{l}<\text { Advantages }> \\
\text {-Various solvents (both high and low b.p) } \\
\text {-Highly conductive (no loss of conductivity) } \\
\text {-Simple, fast, and conducive to mass production }\end{array}$ & $\begin{array}{l}\text { This } \\
\text { work }\end{array}$ \\
\hline
\end{tabular}


sensor array. The highly conductive solvent-exchanged PEDOT:PSS developed in this study could facilitate new opportunities for flexible electronic devices, and the approach could be implemented as a universal technique for solvent exchange of various materials.

\section{Materials and methods}

\section{Solvent exchange process for PEDOT:PSS solutions}

A novel PEDOT:PSS solvent exchange process was carried out in a closed laboratory environment. Initially, a water-based PEDOT:PSS solution (Clevios PH 1000, Heraues) and an organic exchange solvent (1:1 volume ratio) were loaded in a lab-made filtration tank. Then, rotation and ultrafiltration processes were started simultaneously. The ultrafiltration was maintained at a pressure of 4-5 bar by an air-pressure pump. Regenerated cellulose membranes (nominal molecular weight limit: $10 \mathrm{kDa}$ ) were used for this ultrafiltration. The hydrophilic, tight microstructure of the membrane ensured the highest possible permeability with the lowest possible adsorption of PEDOT:PSS gel particles. Once the solvent was exchanged by a factor of $50 \%$, another equivalent amount of solvent was added. This cyclical process was repeated 5-6 times until the solvent exchange ratio was $95 \%$ or higher.

\section{Preparation of a PDMS substrate with a micropyramid array}

Initially, a silicon mold was prepared using standard conventional photolithography procedures, as reported previously ${ }^{20}$. Then, the Si mold surface was cleaned and treated with a 3-amino-propyl triethoxysilane (99\%, Sigma-Aldrich) solution to facilitate the formation of a thin hydrophobic layer for easy removal of the PDMS layer. Pouring the PDMS mixture (base:curing agent = 10:1) on the mold followed by exposure to vacuum allowed PDMS microstructures to form without any bubbles. Later, a flexible polyimide film was placed over the PDMS mixture and pressed with a known weight to promote the desired PDMS thickness throughout the experiments, which was followed by curing at $100^{\circ} \mathrm{C}$ for $20 \mathrm{~min}$, enabling the formation of 3D microstructures that were elastic in nature. Finally, a pyramid PDMS array was obtained by slowly stripping the film off the silicon mold.

\section{Preparation of solvent-exchanged PEDOT:PSS films on the micropyramid array}

The PDMS substrate with the micropyramid array was treated with $\mathrm{O}_{2}$ plasma for $3 \mathrm{~min}$. Then, the solventexchanged PEDOT:PSS solution with EtOH as a solvent was mixed with $5 \mathrm{wt} \%$ dimethyl sulfoxide (DMSO) (99.9\%, Sigma-Aldrich) to enhance the conductivity and diluted twice with EtOH (99.9\%, OCI Company Ltd.) to ensure uniform thin film formation. The mixture was stirred and sonicated for $30 \mathrm{~min}$ and $2 \mathrm{~min}$, respectively, followed by filtration through a $5.0 \mu \mathrm{m}$ nylon syringe filter. Then, the mixture was drop-cast over the pretreated PDMS surface, with the excess solution removed by tilting the substrate vertically. Later, the sample was annealed in a convection oven at $150^{\circ} \mathrm{C}$ for $15 \mathrm{~min}$. The size of the sample with the micropyramid array was $1.5 \mathrm{~cm} \times 1.5 \mathrm{~cm}$.

\section{Fabrication of a resistive pressure sensor device}

The PEDOT:PSS film coating the PDMS pyramid array and Au-deposited PET film served as the bottom and top electrodes. Then, external wires were connected to the top electrode with the aid of anisotropic conductive film (ACF) bonding, while the bottom electrode was attached to the PEDOT:PSS coating with silver paste. Next, the top Au-coated PET film was placed and aligned over the PDMS microstructure, with the electrodes facing each other to further carry out electrical and mechanical characterizations.

\section{Characterization and measurement}

X-ray photoelectron spectroscopy (XPS) was performed with a K-alpha spectrometer (Thermo Fisher Scientific Inc.). Atomic force microscopy (AFM) and scanning electron microscopy (SEM) with energy dispersive X-ray (EDX) spectroscopy images were obtained using a Nanoscope Multimode Iva (Bruker Corp.) and a JEOL JSM-6710F field emission scanning electron microscope, respectively. The conductivities of PEDOT:PSS films were calculated based on their sheet resistance and thickness. The $\mathrm{pH}$ values of solvent-exchanged PEDOT:PSS solutions were measured using an MP-220 pH meter (Mettler-Toledo AG 8603 Schwerzenbach, Switzerland). The contact angles (CA) of PEDOT:PSS solutions were measured by a CA measuring device (Phoenix 300, South Korea). In the CA test solutions, $5 \%$ DMSO or $1 \%$ wetting agent (Capstone FS-30, Chemours Co.) was added to the PEDOT: PSS solutions with water and EtOH solvents, respectively. $\mathrm{I}-\mathrm{V}$ measurements of the resistive pressure sensor device were carried out using a Keithley 4200-SCS semiconductor characterization system (Tektronix Co.). Pressure was applied or released by adding different loads to the device or removing them, respectively. The active area of the pressure sensor was $1.5 \mathrm{~cm} \times 1.5 \mathrm{~cm}$. For a touch panel array application, the solvent-exchanged PEDOT:PSS film coated on the PDMS pyramid array and the Au-deposited PET film were laser patterned by a programmable benchtop pulsed laser system (BasiCube 10, Scanlab Co.).

\section{Results and discussion}

Solvent exchange process using the ultrafiltration method

The important factor in solvent exchange of PEDOT: PSS is that PEDOT:PSS should remain wetted with the 
solvent to prevent coagulation of PEDOT:PSS. For this, a solvent exchange process using the ultrafiltration method was designed. Figure 1a shows the novel solvent exchange process (SEP) by ultrafiltration. The principle of this solvent exchange process is similar to changing cold water to hot water. Similar to adding hot water while removing cold water, an organic solvent well mixed with water is first added to aqueous PEDOT:PSS solution of the same volume. If half of the mixed solvent is removed through the membrane filter, then a solvent exchange ratio of 50\% is obtained. In this mixture, half the volume is water, and the other half is the organic solvent. When this method is repeated 5-6 times, the solvent exchange ratio reaches 95\% or higher. During this solvent exchange process, excess PSS or impurities can also be removed through the membrane filter at the same time. Figure $1 \mathrm{~b}$ shows the dispersion stability of PEDOT:PSS solutions solventexchanged from water to various solvents after the SEP. $\mathrm{EtOH}$ and ethylene glycol (EG) were particularly selected as solvents for the solvent exchange of water-based PEDOT:PSS solutions because $\mathrm{EtOH}$ is widely used in alcohol organic solvents and EG is one of the most widely used conductivity enhancers of PEDOT:PSS. The dispersion stability was excellent in EtOH and EG, which can be explained by the Hansen solubility parameter. PEDOT and PSS have coulombic interactions with each other. The sulfonic acid of PSS is highly hydrophilic and dissociates into $\mathrm{SO}^{-}$and $\mathrm{H}^{+}$in water. Thus, PEDOT:PSS has excellent dispersibility in water due to the strong hydrogen bonding between the water molecules and SO3Supplementary Fig. 2a shows a graph of the Hansen solubility parameters of the solvents used in the SEP, indicating that the index determining the solubility is composed of three forces: the dispersion force $\left(\partial_{\mathrm{D}}\right)$, dipole-dipole force $\left(\partial_{\mathrm{P}}\right)$, and hydrogen bonding force $\left(\partial_{\mathrm{H}}\right)^{21-23}$. Supplementary Table S1 shows the values of $\partial_{\mathrm{D}}$, $\partial_{\mathrm{P}}$, and $\partial_{\mathrm{H}}$ for each solvent. Among these three parameters, $\partial_{\mathrm{H}}$ is mostly related to the dispersion stability of PEDOT:PSS. The higher the $\partial_{\mathrm{H}}$ value of the solvent is, the better PEDOT:PSS is dispersed in the solvent, as shown in Fig. 1b. This relates to the mechanism by which PEDOT: PSS is dispersed in the solvent. PEDOT and PSS are connected by coulombic interactions, and PEDOT:PSS can be dispersed in solvent by the hydrogen bonding force between PSS and the solvent. Therefore, the higher the $\partial_{\mathrm{H}}$ value of the solvent is, the stronger the hydrogen bonding

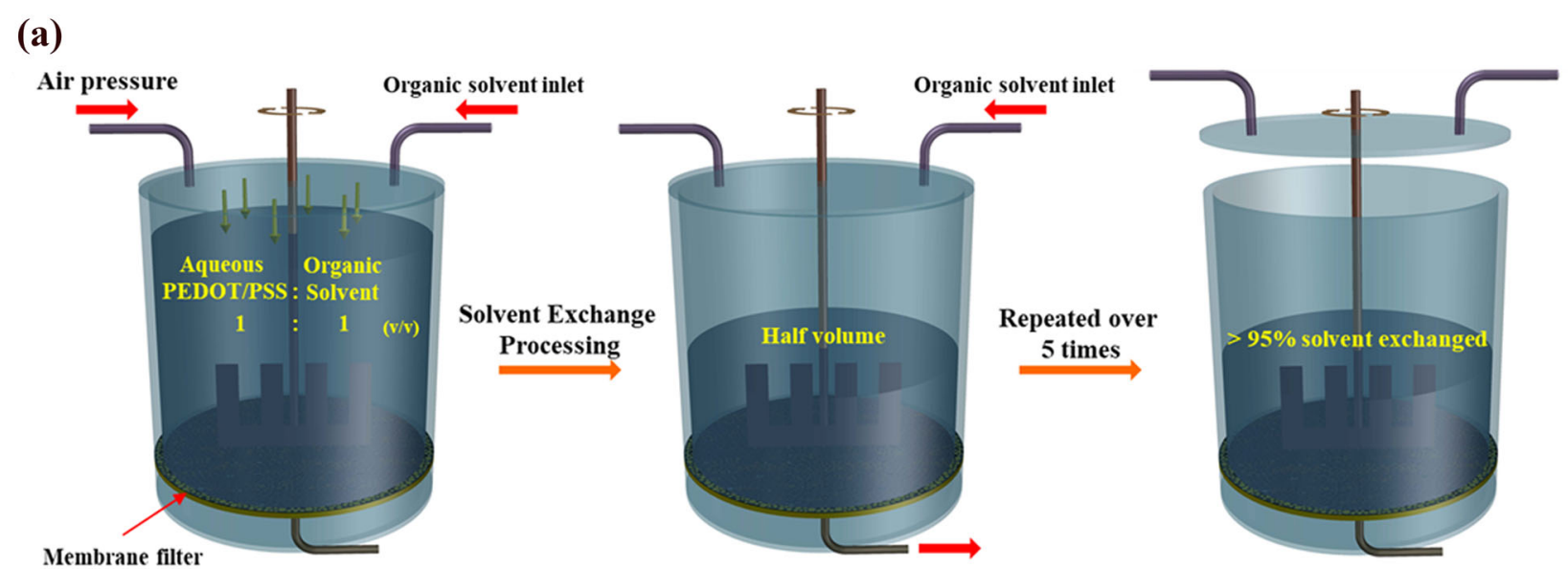

Water + organic solvent + Excess PSS + Impurities

(b)

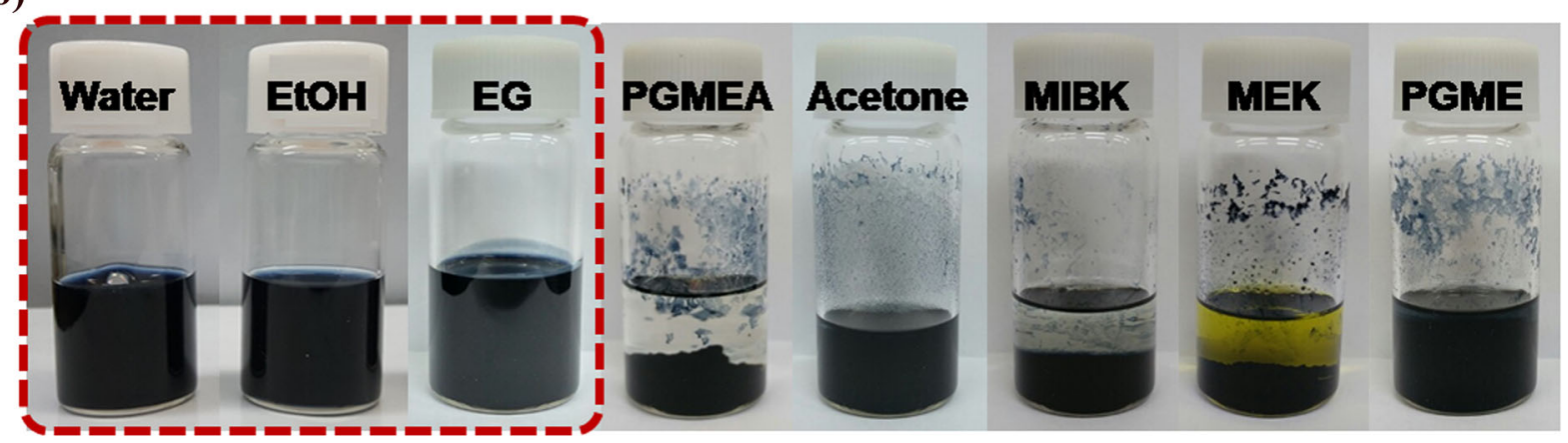

Fig. 1 a Novel solvent exchange process by ultrafiltration. b Dispersion stability of PEDOT:PSS in various solvents after SEP. 
(a)

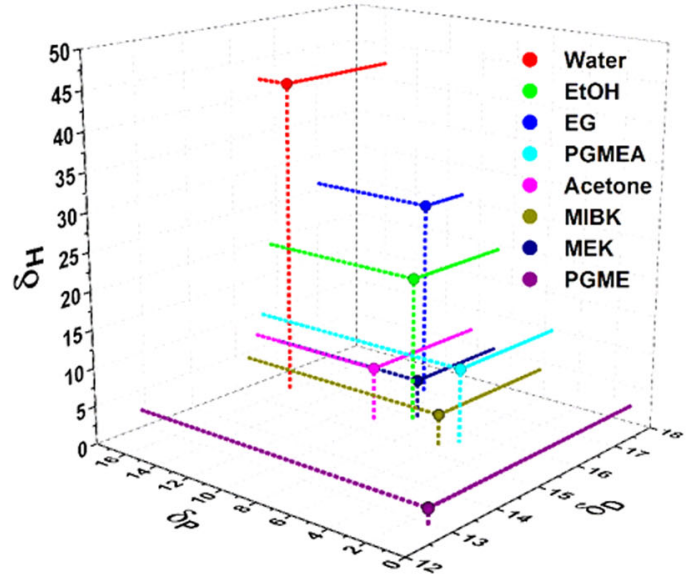

(b)

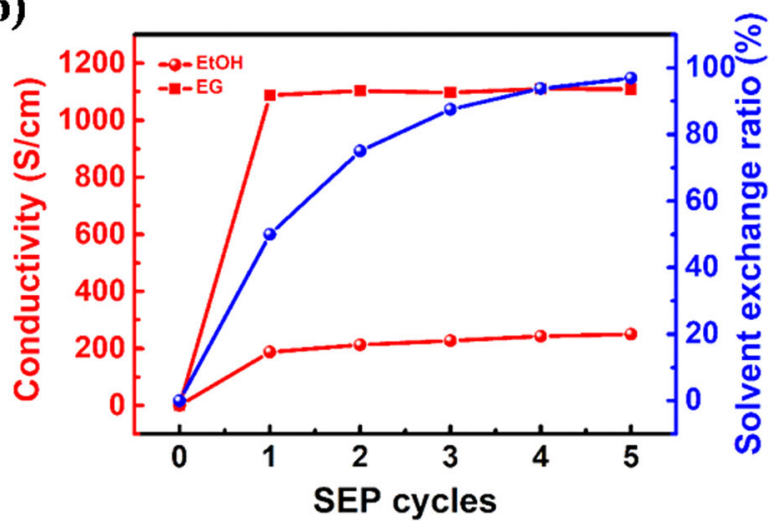

(c)

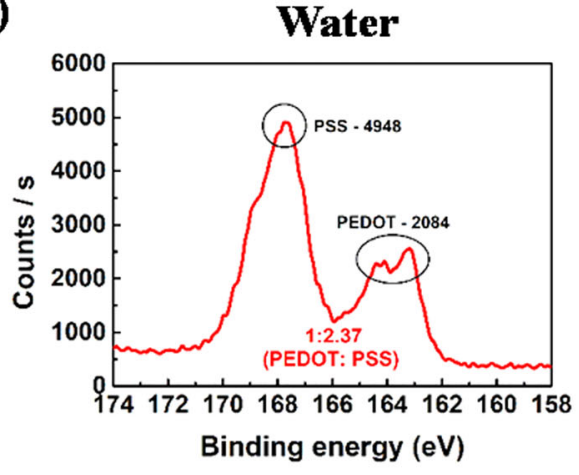

EtOH

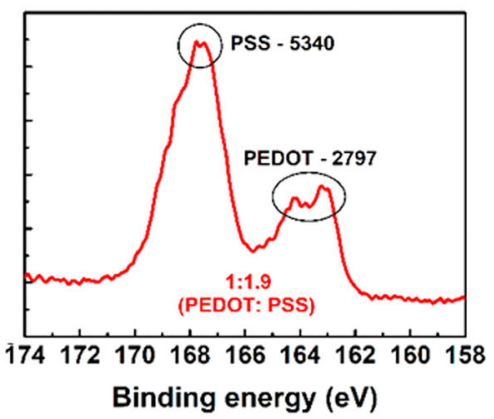

EG

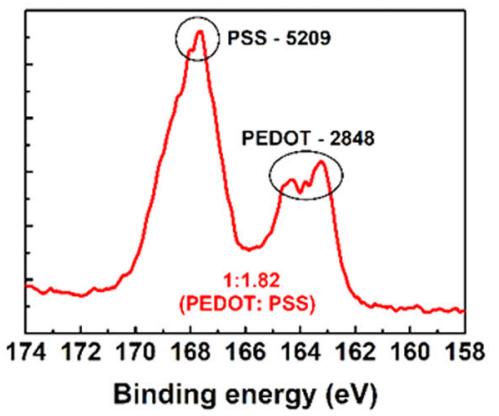

(d)
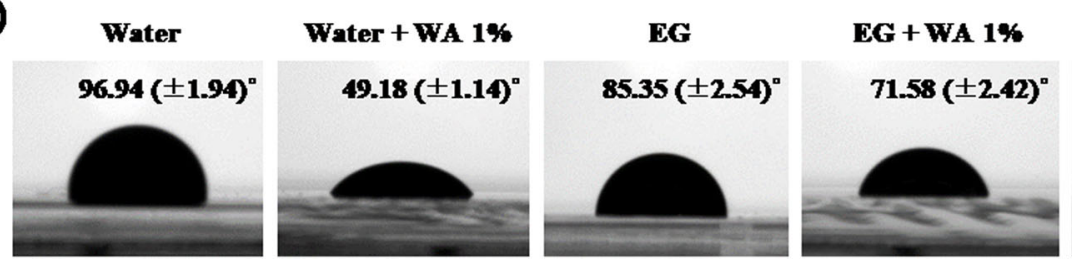

EtOH

$34.67( \pm 1.66)$

EtOH + WA $1 \%$

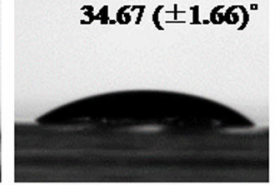

$34.51( \pm 0.86)^{\prime \prime}$

Fig. 2 a Graph of Hansen solubility parameters of the solvents used in SEP. $\mathbf{b}$ Variation in conductivity without the addition of DMSO and solvent exchange ratio depending on the number of SEP cycles. $\mathbf{c}$ XPS spectra of S $2 p$ and (d) contact angle of PEDOT:PSS for various solvents by SEP.

force between the solvent and PSS, so PEDOT:PSS can be more stably dispersed in a solvent with a high $\partial_{\mathrm{H}}$ value. Through our solvent exchange process, it has been confirmed that PEDOT:PSS was very well dispersed in a solvent with a $\partial_{\mathrm{H}}$ value of 19.4 or higher. The solvent exchange ratio and the conductivity without the addition of DMSO are presented in Fig. 2b. After five or more SEP steps, the solvent exchange ratio reached $95 \%$ or higher. Note that the conductivity increased from 0.94 to $250 \mathrm{~S} /$ $\mathrm{cm}$ (in $\mathrm{EtOH}$ ) without the addition of DMSO as a conductivity enhancer. This conductivity enhancement is due to the better connection between the conductive PEDOT chains, as the PEDOT and PSS are segregated by polar solvents such as EtOH ${ }^{24-26}$. Since EG is a typical conductivity enhancer, it exhibits a high conductivity of $1108 \mathrm{~S} / \mathrm{cm}$, even without DMSO. After the addition of
DMSO, the conductivity in the water, EtOH, and EG solvents reached similar maximum values (Table S2). This means that the main chain of PEDOT, which is primarily responsible for the conductivity, remains unchanged, even after the SEP. XPS analysis was performed to confirm the change in the ratio between PEDOT and PSS after the SEP. Supplementary Fig. 2c shows XPS spectra of S 2p of PEDOT:PSS films from the water, EtOH, and EG solvents. The PEDOT:PSS ratio decreased from 1:2.37 in water to 1:1.90 in $\mathrm{EtOH}$ and 1:1.82 in EG, demonstrating the effect of removing the residual PSS in water. As the highly acidic PSS was removed, the $\mathrm{pH}$ of the PEDOT:PSS solution increased from 2.4 in water to 4.5 in EtOH and 4.4 in EG (Supplementary Table S2). Since the degradation of the atmospheric stability of PEDOT:PSS is mainly due to the hygroscopic property of PSS $^{27}$, the reduction of the excess 
PSS can improve the atmospheric stability of PEDOT:PSS. Thus, the atmospheric stability of PEDOT:PSS depending on the solvent of PEDOT:PSS was analyzed by measuring the sheet resistance over time when exposed to air (temperature: $25^{\circ} \mathrm{C}$, relative humidity: $30 \%$ ). The sheet resistance of the aqueous PEDOT:PSS increased by $98 \%$ on day 7, while the sheet resistance of EtOH- or EGexchanged PEDOT:PSS increased by 38 or $34 \%$, respectively. In other words, the atmospheric stability of solvent-exchanged PEDOT:PSS was improved by nearly three times compared to the aqueous PEDOT:PSS due to the effect of removing excess PSS (Supplementary Fig. S1). Supplementary Fig. S2a, b shows SEM and AFM images, implying that the gel particles increase in size after solvent exchange and that the crystallinity is improved by the 3D network structure and $\pi-\pi$ stacking. This phenomenon is particularly evident in EG and is similar to that in previous reported studies of PEDOT:PSS treated by polar solvents, including EtOH and $\mathrm{EG}^{28-33}$. The contact angles (CA) of PEDOT:PSS solutions depending on the solvent and addition of a wetting agent (WA) on PDMS are presented in Fig. 2d (Supplementary Table S3). Among all the CA measurements, the PEDOT: PSS solution with water as a solvent showed a large variation from $96.94^{\circ}$ to $49.18^{\circ}$, while the PEDOT:PSS solution with the EG solvent showed $\sim 14^{\circ}$ less variation, and the PEDOT:PSS solution with the EtOH solvent exhibited a negligible change $\left(\sim 0.16^{\circ}\right)$ after the addition of the WA at $1 \%$. Although the PEDOT:PSS solution with water exhibited a lower CA after the addition of the WA $\left(49.18^{\circ}\right)$, the PEDOT:PSS solution with the EtOH solvent without

the WA displayed an even lower CA, thereby avoiding the necessity of a WA for wetting the surface. Therefore, it is clear from Fig. 2d that the PEDOT:PSS solution with $\mathrm{EtOH}$ as a solvent without a WA exhibited better coating properties (lower CA) than the solutions with EG and water. It is noteworthy that only the solutions with water and $\mathrm{EtOH}$ were mixed with 5\% DMSO, not the solution with EG, because EG itself is a conductivity enhancer.

\section{Fabrication and characterization of a pressure sensor based on EtOH solvent-exchanged PEDOT:PSS}

A step-by-step pressure sensor fabrication process using solvent-exchanged PEDOT:PSS with EtOH as a sensing material is schematically illustrated in Fig. 3a. The corresponding multilayer design layout and the sensor attached to the wrist artery for blood pulse monitoring are presented in Fig. 3b. A schematic illustration of the circuit model of the sensor mechanism with and without pressure is shown in Fig. 3c, where $R_{1}$ and $R_{2}$ represent the resistance before and after pressure is applied, respectively. To ensure PDMS microstructure formation, SEM measurements were performed, and corresponding images were obtained (Supplementary Fig. S3a-d). It was concluded that all pyramidal microstructures form sharp edges at the top without any structural deformation. To ensure a uniform coating of PEDOT:PSS on the uneven microstructure, drop-casting was preferred instead of spin-coating, which led to a nonuniform coating. As a systematic study, initially, pristine PEDOT:PSS solventexchanged with $\mathrm{EtOH}$ without dilution was used as prepared, without any additional changes. In this case, the SEM images showed thick coatings with a nonuniform thickness over the pyramids, as shown in Supplementary Fig. S4a,b. To obtain a more uniform and thin coating, the pristine solution was diluted twice with the same solvent (EtOH). Uniform and conformal nanocoating of PEDOT:

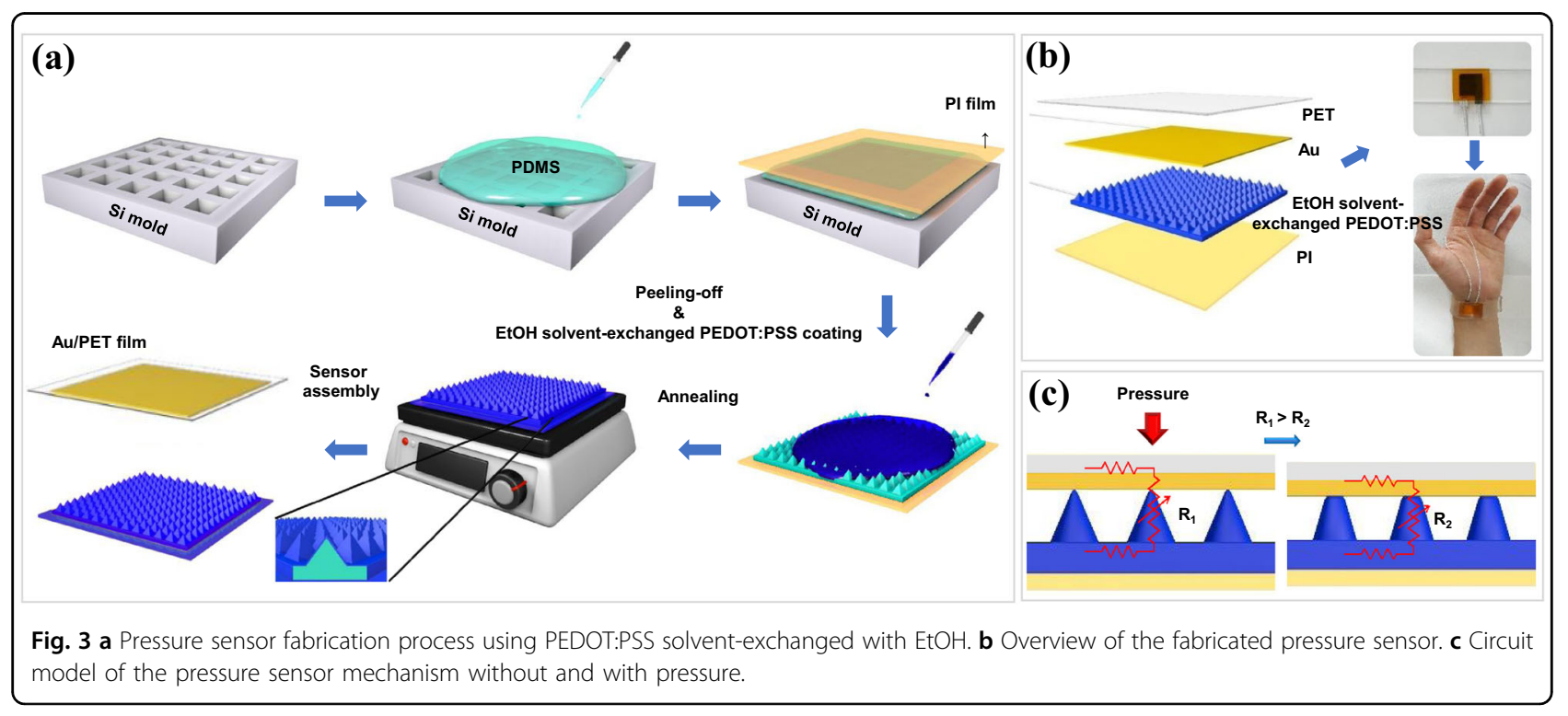




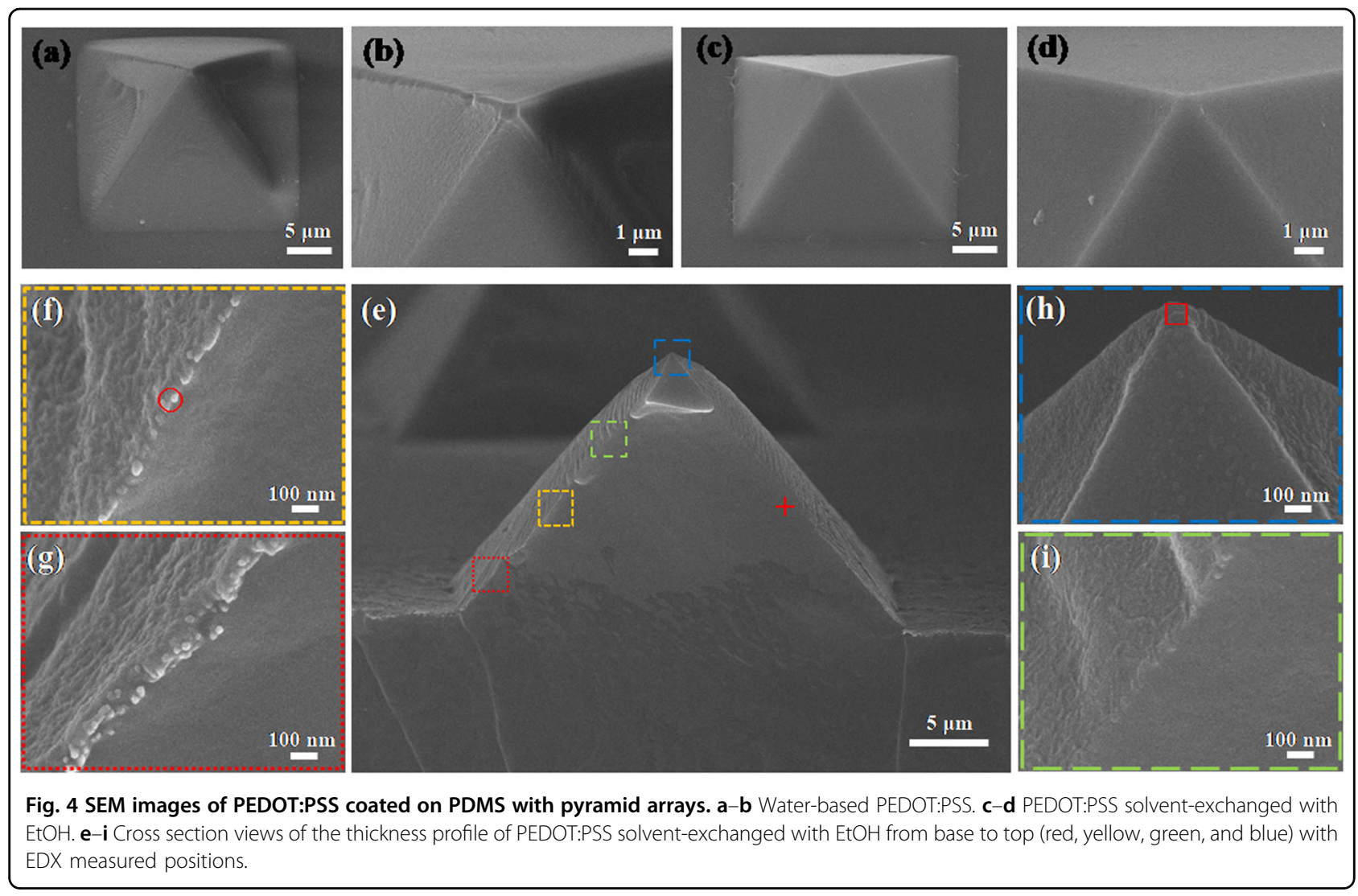

PSS on the uneven surface without any agglomeration was observed, which is essential for practical applications (Fig. 4c, d). To further investigate the nanolevel thickness, uniformity, and presence of the PEDOT:PSS coating over the pyramids, SEM and EDX measurements were performed. As expected, due to the inclined surface, the thickness profile from the base to the top of a pyramid decreased from 90 to $10 \mathrm{~nm}$ according to cross-sectional SEM images, as shown in Fig. 4e-i. Similarly, the presence of the PEDOT:PSS coating was confirmed by EDX at the respective positions shown in Fig. 4e, $\mathrm{f}, \mathrm{h}$ and Supplementary Table S4. The conformation and particle size $(\approx 30 \mathrm{~nm})$ of PEDOT:PSS are consistent with previous studies ${ }^{34,35}$. Notably, sharp edges with a uniform and conformal coating were observed at the top of the pyramids (also confirmed by EDX), as shown in Fig. 4h, which are crucial for minimizing large resistance deviations in response to pressure in the low pressure regime. On the other hand, commercial waterbased PEDOT:PSS partially coated near the edge of the PDMS structure, leading to poor sensing performance (Figs. 4a, b and 5b). This can also be seen in Fig. 2d; the water-based PEDOT:PSS solution exhibited a high CA compared to the EtOH-based solution.

The PEDOT:PSS solvent-exchanged with EtOH (diluted twice with EtOH) coating the PDMS pyramidal microstructure array was considered as a sensing material for the other electrical and mechanical characterizations. I-V measurements were carried out by applying a voltage across the two (top and bottom) electrodes such that a current flowed from the flat PEDOT:PSS substrate to the flat $\mathrm{Au}$ substrate through the pyramid tops. As pressure was applied to the top electrode, the pyramidal structures deformed, thereby increasing the contact area and, as a result, decreasing the resistance. Further increasing the pressure led to a negligible change in resistance due to the limited deformation of the pyramid microstructures. Conversely, as the pressure was decreased, due to the elastic nature, the PDMS structure tended to return to its original position, causing the resistance to increase. Figure $5 \mathrm{a}, \mathrm{b}$ presents the sensitivity of the proposed pressure sensor, defined as $S=\left(\Delta R / R_{0}\right) / \Delta P$, where $\Delta R$ is the change in resistance under applied pressure $P$, and $R_{0}$ is the initial resistance under zero pressure. In the case of water-based PEDOT:PSS, the sensor showed poor sensitivity with large hysteresis, which is unfavorable for pressure sensing applications. In contrast, the sensor with PEDOT:PSS solvent-exchanged with $\mathrm{EtOH}$ (diluted twice with $\mathrm{EtOH}$ ) showed high sensitivity with excellent reliability upon the increase and decrease (from 0 to $1 \mathrm{kPa}$ and $1 \mathrm{kPa}$ to $0 \mathrm{~Pa}$ ) of pressure, almost retaining the original values when the pressure was removed, as shown in Fig. 5b. The plot exhibited two distinct pressure sensitivity regimes, one for 
(a)

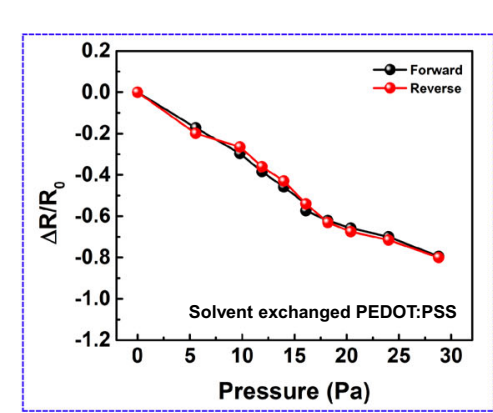

(b) 0.

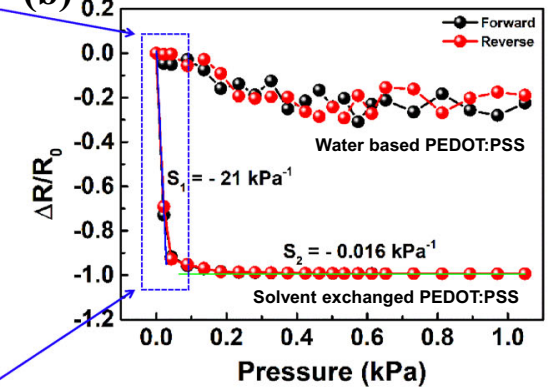

(c)

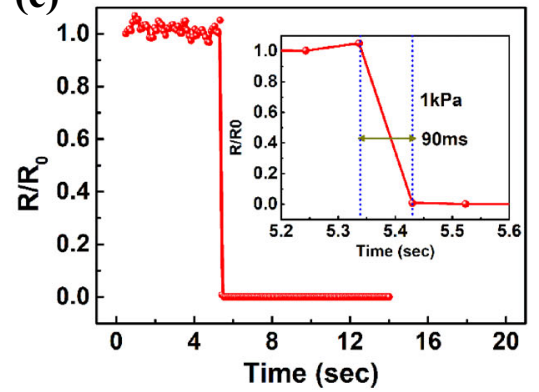

(d)
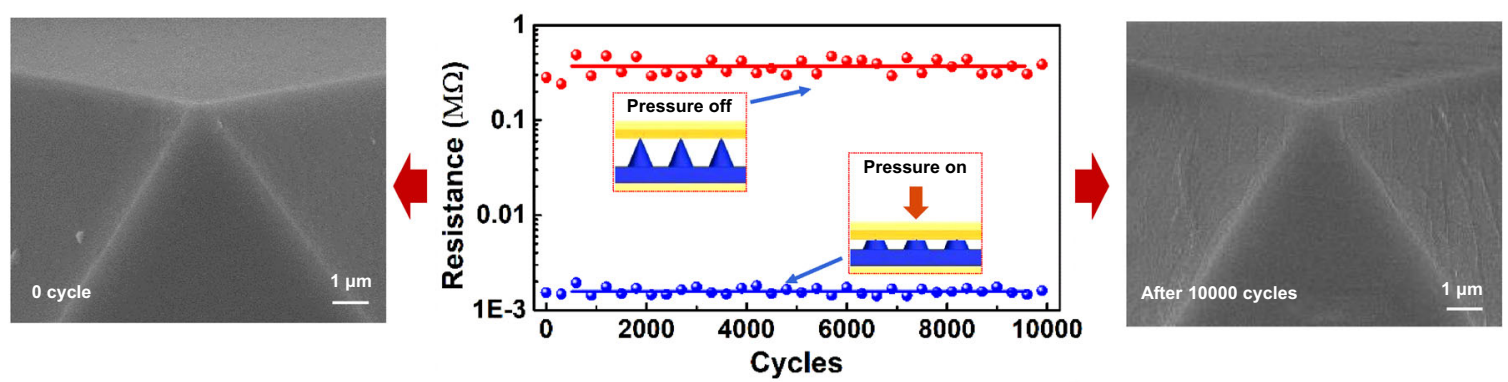

(e)

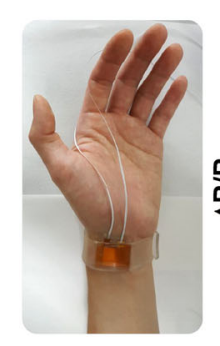

(f)

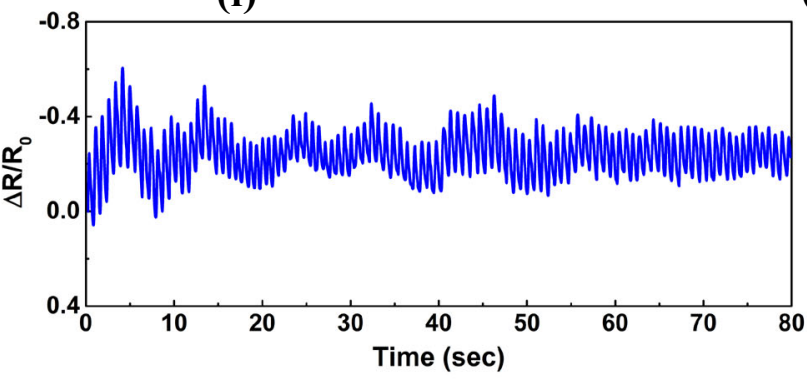

(g)

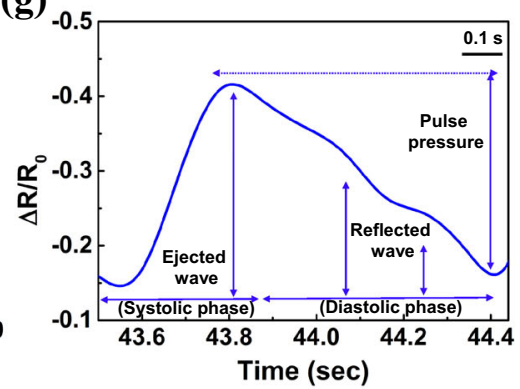

(h)

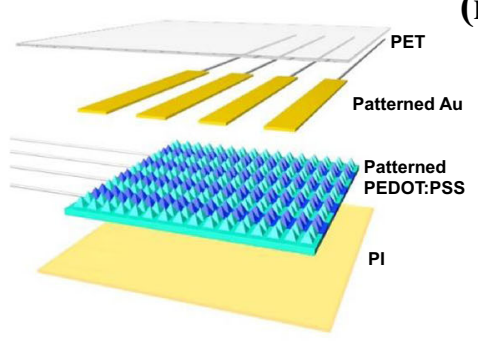

(i)

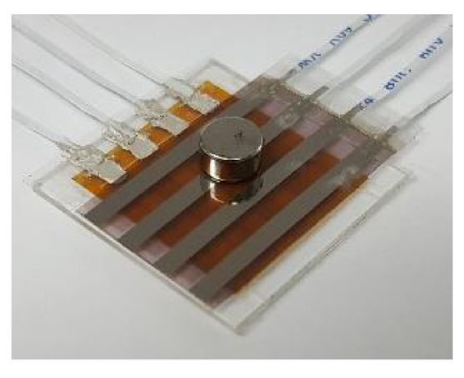

(j)

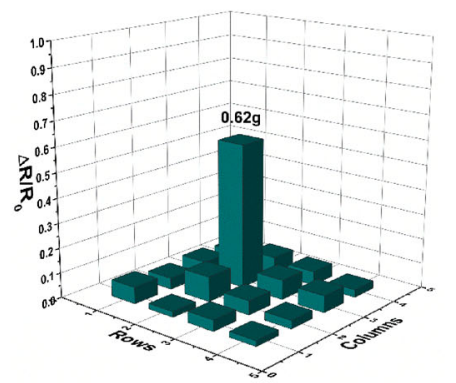

Fig. $\mathbf{5}$ a, b Pressure sensitivity in the linear and saturation regime and (c) Response time of the proposed pressure sensor using PEDOT:PSS solvent-exchanged with EtOH. d Mechanical stability over 10000 cycles. Application of the pressure sensor to measure the human pulse wave $(\mathbf{e}-\mathbf{g})$ Sensor wrapped on the wrist of the hand, blood pulse rate measured and pulse showing systolic and diastolic phase. $\mathbf{h}-\mathbf{j}$ Pressure sensor array demonstration.

low-pressure values $<100 \mathrm{~Pa}$, with a sensitivity $S 1$ of $-21 \mathrm{kPa}^{-1}$, and the other for medium pressure values of $0.1-1 \mathrm{kPa}$, with a sensitivity $S 2$ of $-0.016 \mathrm{kPa}^{-1}$. Note that the PEDOT:PSS solvent-exchanged with $\mathrm{EtOH}$ is not coated thinly and uniformly without dilution, and the pressure sensitivity is very poor, with a sensitivity S0 of $-0.0001 \mathrm{kPa}^{-1}$ (Supplementary Figs. S4, S5). The performance of the proposed sensor was compared with that of other sensors reported in the literature, as summarized in Supplementary Table S5 ${ }^{36-47}$. Although a few reports demonstrate very high sensitivities $>20 \mathrm{kPa}^{-1}$, the procedures to fabricate these sensors are very complex, and the structures obtained by nonconventional techniques are inadequate for producing reliable and repeatable data. Depending on the designed parameters of the microstructures, such as the shape, width, height, and spacing, 
the sensitivity and pressure regimes vary ${ }^{20,47-49}$. Our sensor showed a fast response time of only $90 \mathrm{~ms}$, with a maximum load of $1 \mathrm{kPa}$, which is essential for practical pressure sensor applications (Fig. 5c). Figure 5d presents the mechanical stability of the pressure sensor using PEDOT: PSS solvent-exchanged with EtOH. Even after applying a cyclic pressure of $0.3 \mathrm{kPa}$ for 10,000 cycles, the sensor exhibited high mechanical stability without peeling-off or resistance change.

\section{Application of the proposed pressure sensor}

Figure $5 \mathrm{e}-\mathrm{g}$ presents the blood pulse and pressure sensor array applications of the proposed pressure sensor. To demonstrate a blood pulse rate sensor, the sensor was fabricated by laminating the PEDOT:PSS-coated PDMS microstructures with face-to-face alignment of the $\mathrm{Au}$ coated PET sheet, followed by extracting external wires from the edges of the films with the aid of ACF bonding. Among various vital signs of the human body, the pulse is considered a primary vital sign of the human body to prevent cardiac diseases. The proposed pressure sensor attached to a wrist showed pulse waveforms in real-time monitoring, with a pulse frequency of 78 beats $/ \mathrm{min}$, which corresponds to that of a healthy adult. Moreover, the sensor exhibited distinguishable systolic and diastolic phases with $\mathrm{P}_{1}, \mathrm{P}_{2}$, and $\mathrm{P}_{3}$ peaks indicating incident, systolic, and diastolic waves ${ }^{4}$. Furthermore, Fig. $5 \mathrm{~h}-\mathrm{j}$ shows a $4 \times 4$-pixel array pressure mapping panel that enabled spatial resolution of the pressure. Figure $5 \mathrm{~h}$ shows the multilayer structure of the array sensor. The PEDOT:PSScoated pyramidal PDMS structure was patterned by a benchtop programmable nanosecond pulse laser to form four electrodes side-by-side in a row. Similarly, the Aucoated PET was also patterned to form four electrodes in a column (Supplementary Fig. S6). The four formed electrodes were connected to external wires with the aid of ACF bonding, while the patterned PEDOT:PSS layer was connected with silver paste. Then, the array sensor was sandwiched by attaching the top electrodes perpendicular to the bottom electrodes. The load of $0.6 \mathrm{~g}$ corresponded to $1.5 \mathrm{kPa}$ applied to the second row and third column of the sensor array, and the difference in the applied pressure of the pixel was observed via the increased magnitude of the resistance, presented in Fig. $5 \mathrm{~h}$, i. Simultaneously, the spatial pressure distribution on pixels other than the applied pressure pixel exhibited a lower magnitude, thereby enabling precise localization of the applied load. This demonstrates the viability of the proposed pressure sensor for obtaining spatial pressure distributions.

\section{Conclusions}

Herein, highly conductive solvent-exchanged PEDOT: PSS dispersed in EtOH or EG showed very stable and homogeneous solutions. The PEDOT:PSS solution with $\mathrm{EtOH}$ as a solvent showed better coating properties than water without any aggregates. Applying the $\mathrm{EtOH}$-based conductive PEDOT:PSS solution coating on hydrophobic substrates, such as PDMS, avoids the necessity of wetting agents, such as surfactants that alter the original properties of the materials. The pressure sensor fabricated by nanocoating conductive solutions on pyramidal PDMS structures exhibited ultrahigh sensitivity with a stable sensing performance. Moreover, the blood pulse rate and pressure mapping array sensors produced here were very accurate and sensitive, enabling spatial resolution of pressure. The advantages exhibited by these physical and mechanical properties of the solvent-exchanged PEDOT: PSS on microstructures enable potential applications in human-machine interfaces.

\begin{abstract}
Acknowledgements
This research was supported by the Basic Science Research Program through the National Research Foundation of Korea (NRF) (2017R1A2B4009963, 2018R1D1A1B07048232, 2019R111A1A01059217, and 2020R111A1A01073884 funded by the Ministry of Education). The researchers also acknowledge the support of the Korea Institute of Industrial Technology (KITECH); Research Project of Core Technology for Industry Leading Manufacture (KITECH EO-200019) and Korea Evaluation Industrial Technology (KEIT) through the Industrial Strategic Technology Development Program (No. 10079571).
\end{abstract}

\section{Author contributions \\ J-J.L. and S-G. mainly performed the experiment and wrote the paper. B-J.L., S-J.K. and S-Y.K. supported the experiment. S-J.L. and S-K.K. managed and advised on this research.}

\section{Conflict of interest}

The authors declare that they have no conflict of interest.

\section{Publisher's note}

Springer Nature remains neutral with regard to jurisdictional claims in published maps and institutional affiliations.

Supplementary information is available for this paper at https://doi.org/ 10.1038/s41427-020-00238-z.

Received: 10 January 2020 Revised: 11 June 2020 Accepted: 18 June 2020. Published online: 18 September 2020

\section{References}

1. Someya, T. et al. A large-area, flexible pressure sensor matrix with organic fieldeffect transistors for artificial skin applications. Proc. Natl Acad. Sci. 101, 9966-9970 (2004).

2. Chou, H.H. et al. chameleon-inspired stretchable electronic skin with interactive colour changing controlled by tactile sensing. Nat. Commun. 6, 8011 (2015).

3. Boutry, C. M. et al. A hierarchically patterned, bioinspired e-skin able to detect the direction of applied pressure for robotics. Sci. Robot. 3, eaau6914 (2018).

4. Park, J., Kim, M., Lee, Y., Lee, H. S. \& Ko, H. Fingertip skin-inspired microstructured ferroelectric skins discriminate static/dynamic pressure and temperature stimuli. Sci. Adv. 1, e1500661 (2015).

5. Meer, S., Kausar, A. \& lqbal, T. Trends in conducting polymer and hybrids of conducting polymer/carbon nanotube: a review. Polym. Plast. Technol. Eng. 55, 1416-1440 (2016).

6. Zhan, C. et al. Conductive polymer nanocomposites: a critical review of modern advanced devices. J. Mater. Chem. C 5, 1569-1585 (2017). 
7. Mehmood, U., Al-Ahmed, A. \& Hussein, I. A. Review on recent advances in polythiophene based photovoltaic devices. Renew. Sustain. Energy Rev. 57, 550-561 (2016).

8. Meng, Q., Cai, K., Chen, Y. \& Chen, L. Research progress on conducting polymer based supercapacitor electrode materials. Nano Energy 36, 268-285 (2017).

9. Lee, J. J., Lee, S. H., Kim, F. S., Choi, H. H. \& Kim, J. H. Simultaneous enhancement of the efficiency and stability of organic solar cells using PEDOT:PSS grafted with a PEGME buffer layer. Org. Electron. 26, 191-199 (2015).

10. Lee, J. J., Yoo, D., Park, C., Kim, J. H. \& Choi, H. H. Behavior of toxic gas sensor based on conducting polypyrrole. Macromol. Symp. 354, 280-286 (2015).

11. Yoo, D., Lee, J. J., Park, C., Choi, H. H. \& Kim, J.-H. N-type organic thermoelectric materials based on polyaniline doped with the aprotic ionic liquid 1-ethyl-3methylimidazolium ethyl sulfate. RSC Adv. 6, 37130-37135 (2016).

12. Lee, J. J., Yoo, D., Park, C., Choi, H. H. \& Kim, J. H. All organic-based solar cell and thermoelectric generator hybrid device system using highly conductive PEDOT: PSS film as organic thermoelectric generator. Sol. Energy 134, 479-483 (2016).

13. Lindim, C., van Gils, J. \& Cousins, I. T. Europe-wide estuarine export and surface water concentrations of PFOS and PFOA. Water Res. 103, 124-132 (2016).

14. Supreeyasunthorn, P., Boontanon, S. K. \& Boontanon, N. Perfluorooctane sulfonate (PFOS) and perfluorooctanoic acid (PFOA) contamination from textiles. J. Environ. Sci. Heal. Part A 51, $472-477$ (2016).

15. Kang, J. S., Ahn, T.-G. \& Park, J.-W. Perfluorooctanoic acid (PFOA) and perfluooctane sulfonate (PFOS) induce different modes of action in reproduction to Japanese medaka (Oryzias latipes). J. Hazard. Mater. 368, 97-103 (2019).

16. Seshadri, V. Replacing aoueous with non-aqueous solvent (2012).

17. Kim, J. Y., Jung, J. H., Lee, D. E. \& Joo, J. Enhancement of electrical conductivity of poly(3,4-ethylenedioxythiophene)/poly(4-styrenesulfonate) by a change of solvents. Synth. Met. 126, 311-316 (2002).

18. Shiraishi, K., Kanbara, T., Yamamoto, T. \& Groenendaal, L. Bert. Preparation of a soluble and neutral alkyl derivative of poly(3,4-ethylene-dioxythiophene) and its optical properties. Polymer 42, 7229-7232 (2001).

19. Charba, A. et al. Preparation of water-free PEDOT dispersions in the presence of reactive polyisoprene stabilizers. Langmuir 30, 12474-12482 (2014).

20. Mannsfeld, S. C. B. et al. Highly sensitive flexible pressure sensors with microstructured rubber dielectric layers. Nat. Mater. 9, 859-864 (2010).

21. Andecochea Saiz, C., Darvishmanesh, S., Buekenhoudt, A. \& Van der Bruggen, B. Shortcut applications of the Hansen solubility parameter for organic solvent nanofiltration. J. Memb. Sci. 546, 120-127 (2018).

22. Gårdebjer, S. et al. Using Hansen solubility parameters to predict the dispersion of nano-particles in polymeric films. Polym. Chem. 7, 1756-1764 (2016).

23. Mao, B. et al. Solubility-parameter-guided solvent selection to initiate ostwald ripening for interior space-tunable structures with architecture-dependent electrochemical performance. Angew. Chem. Int. Ed. 57, 446-450 (2018).

24. Alemu, D., Wei, H.-Y., Ho, K.-C. \& Chu, C.-W. Highly conductive PEDOT:PSS electrode by simple film treatment with methanol for ITO-free polymer solar cells. Energy Environ. Sci. 5, 9662 (2012).

25. Xia, Y. \& Ouyang, J. PEDOT:PSS films with significantly enhanced conductivities induced by preferential solvation with cosolvents and their application in polymer photovoltaic cells. J. Mater. Chem. 21, 4927 (2011).

26. Rwei, S.-P., Lee, Y.-H., Shiu, J.-W., Sasikumar, R. \& Shyr, U.-T. Characterization of solvent-treated PEDOT:PSS thin films with enhanced conductivities. Polymer 11, 134 (2019).

27. Fan, X. et al. PEDOT:PSS for flexible and stretchable electronics: modifications, strategies, and applications. Adv. Sci. 6, 1900813 (2019).

28. Kim, Y. H. et al. Highly conductive PEDOT:PSS electrode with optimized solvent and thermal post-treatment for ITO-free organic solar cells. Adv. Funct. Mater. 21, 1076-1081 (2011).
29. Ouyang, J. et al. On the mechanism of conductivity enhancement in poly(3,4 ethylenedioxythiophene):poly(styrene sulfonate) film through solvent treatment. Polymer 45, 8443-8450 (2004).

30. Hu, Z., Zhang, J. \& Zhu, Y. Effects of solvent-treated PEDOT:PSS on organic photovoltaic devices. Renew. Energy 62, 100-105 (2014).

31. Park, H., Shi, Y. \& Kong, J. Application of solvent modified PEDOT:PSS to graphene electrodes in organic solar cells. Nanoscale 5, 8934 (2013).

32. Li, X.Y. et al. The solvent treatment effect of the PEDOT:PSS anode interlayer in inverted planar perovskite solar cells. RSC Adv. 6, 24501-24507 (2016).

33. Zhang, S., Fan, Z., Wang, X., Zhang, Z. \& Ouyang, J. Enhancement of the thermoelectric properties of PEDOT:PSS via one-step treatment with cosolvents or their solutions of organic salts. J. Mater. Chem. A 6, 7080-7087 (2018).

34. Wang, G.-F., Tao, X.-M., Xin, J. H. \& Fei, B. Modification of conductive polymer for polymeric anodes of flexible organic light-emitting diodes. Nanoscale Res. Lett. 4, 613-617 (2009).

35. Ouyang, J. "Secondary doping" methods to significantly enhance the conductivity of PEDOT:PSS for its application as transparent electrode of optoelectronic devices. Displays 34, 423-436 (2013).

36. Pan, L. et al. An ultra-sensitive resistive pressure sensor based on hollowsphere microstructure induced elasticity in conducting polymer film. Nat. Commun. 5, 3002 (2014).

37. Tian, $\mathrm{H}$. et al. A graphene-based resistive pressure sensor with record-high sensitivity in a wide pressure range. Sci. Rep. 5, 8603 (2015).

38. Zhu, B. et al. Microstructured graphene arrays for highly sensitive flexible tactile sensors. Small 10, 3625-3631 (2014).

39. Choong, C.-L. et al. Highly stretchable resistive pressure sensors using a conductive elastomeric composite on a micropyramid array. Adv. Mater. 26, 3451-3458 (2014).

40. Yao, H.-B. et al. A flexible and highly pressure-sensitive graphene-polyurethane sponge based on fractured microstructure design. Adv. Mater. 25, 6692-6698 (2013).

41. Park, J. et al. Giant tunneling piezoresistance of composite elastomers with interlocked microdome arrays for ultrasensitive and multimodal electronic skins. ACS Nano 8, 4689-4697 (2014).

42. Kim, K.-H. et al. Wearable resistive pressure sensor based on highly flexible carbon composite conductors with irregular surface morphology. ACS Appl. Mater. Interfaces 9, 17499-17507 (2017).

43. Lou, C. et al. A graphene-based flexible pressure sensor with applications to plantar pressure measurement and gait analysis. Materials 10, 1068 (2017).

44. Luo, N. et al. Hollow-structured graphene-silicone-composite-based piezoresistive sensors: decoupled property tuning and bending reliability. Adv. Mater. 29, 1702675 (2017).

45. Pan, J., Liu, S., Yang, Y. \& Lu, J. A highly sensitive resistive pressure sensor based on a carbon nanotube-liquid crystal-PDMS composite. Nanomaterials $\mathbf{8}, 413$ (2018).

46. Pang, $Y$. et al. Epidermis microstructure inspired graphene pressure sensor with random distributed spinosum for high sensitivity and large linearity. ACS Nano 12, 2346-2354 (2018).

47. Lee, Y. et al. Flexible ferroelectric sensors with ultrahigh pressure sensitivity and linear response over exceptionally broad pressure range. ACS Nano 12, 4045-4054 (2018).

48. Zhang, Y. et al. Flexible and highly sensitive pressure sensor based on microdome-patterned PDMS forming with assistance of colloid self-assembly and replica technique for wearable electronics. ACS Appl. Mater. Interfaces $\mathbf{9}$, 35968-35976 (2017).

49. Tee, B. C.-K. et al. Tunable flexible pressure sensors using microstructured elastomer geometries for intuitive electronics. Adv. Funct. Mater. 24, 5427-5434 (2014) 